\title{
Electromagnetic Interference Impact of Wireless Power Transfer System on Data Wireless Channel
}

\author{
Elena N. Baikova ${ }^{1}$, Stanimir S. Valtchev ${ }^{1}$, Rui Melício ${ }^{2,3(\sqrt{ })}$, and Vítor M. Pires ${ }^{4}$ \\ ${ }^{1}$ EST FCT, Universidade Nova, Monte da Caparica, Lisbon, Portugal \\ 2 IDMEC/LAETA, Instituto Superior Técnico, Universidade de Lisboa, Lisbon, Portugal \\ ruimelicio@gmail.com \\ 3 Departamento de Física, Escola de Ciências e Tecnologia, \\ Universidade de Évora, Évora, Portugal \\ ${ }^{4}$ EST Setúbal, Instituto Politécnico de Setúbal, Setúbal, Portugal
}

\begin{abstract}
This paper focuses on measurement and analysis of the electromagnetic fields generated by wireless power transfer system and their possible interaction on data transmission channel. To measure the levels of electromagnetic fields and spectrum near the wireless power transfer equipment the measurement system in the frequency range $100 \mathrm{kHz}$ to $3 \mathrm{GHz}$ was used. Due to the advances in technology it becomes feasible to apply the wireless power transfer in the electric vehicles charging. Currently, in the Faculty of Science and Technology of the University Nova high power wireless power transfer systems are in development. Those systems need to be controlled by several microcontrollers in order to optimize the energy transmission. Their mutual communication is of extreme importance especially when high intensity fields will generate highly undesired influence. The controllers are supposed to communicate with each other through radio frequency data channels. The wireless power transfer system with the electromagnetic interference may influence or completely disrupt the communication which will be a severe problem.
\end{abstract}

Keywords: Wireless power transfer . Wireless data transmission . Electromagnetic field $\cdot$ Electromagnetic interference $\cdot$ Experimental results

\section{Introduction}

Wireless power transfer (WPT) is a promising technology which attracts attention of researchers and manufacturers. In the area of low power the applications are already wide spread: laptops, mobile phones, PDA, wireless headphones, implants or razors. In the same time the high power equipment is also eager to get rid of the wires too, e.g. the industry of intelligent machining systems, robots, the forklift trucks, and electric/hybrid cars. Recently the great deal of attention has been focused on the wireless charging systems for electric vehicles (EV) [1,2].

Among the existent WPT technologies one of the best results was obtained by Kurs et al. using magnetically coupled resonators [3]. The magnetic resonance technology is 
proven to be the most suitable to achieve efficient energy transfer for the EV wireless charging [3-5]. The magnetic coupling system is more advantageous since it doesn't need an accurate parking position of the vehicle as in the case of inductive coupling.

Communications play a key role in wireless charging systems. The data transmission during wireless power transfer allows a transmitter unit to detect and identify receivers and optimize the energy transfer process by increasing or decreasing the transferred power. The simultaneous power and data exchange by proper choice of the modulation strategy are proposed in the inductive powering systems [6, 7] and in the resonant systems $[8,9]$.

In this paper, an application of wireless communications technologies in high power WPT systems is discussed focusing on the electromagnetic compatibility problems in simultaneous wireless energy and data transfer system. To the best of authors' knowledge, the wide overview on the electromagnetic compatibility (EMC) problems of wireless power transfer simultaneously with the wireless data transmission has not yet been reported [10], so this paper is the contribution in the field of EMC issues on wireless power and data transmission.

\section{Technological Innovation for Cyber-Physical Systems}

Nowadays there is a global integration increase of renewable energy systems in the existing electric grid, i.e., power system. Renewable technologies are dependent on weather conditions and are demanding regarding integration into the electric grid. This issue that needs to be fully solved involves the development of smart grid systems [11].

Cyber-physical systems (CPS) can be described as smart systems that includes software and hardware, namely components for sensing, monitoring, gathering, actuating, computing, communication and controlling physical infrastructures, completely integrated and directly interacting to sense the alterations in the state of the surrounding environment $[12,13]$. In CPS computing and physical systems interact tightly in realtime. Smart electric grid, smart buildings, healthcare, wireless charging systems for electric vehicles are examples of emerging CPS.

The WPT system for the electric vehicles charging proposed in this paper is controlled by two microcontrollers in order to optimize the energy transmission. The mutual communication between the physical sub-systems, i.e. microcontrollers with sensing components, which allows to control and management of EV charging process, is the example of CPS as an integration of computation/communication with physical processes. In this case the bi-directional data transmission through a Radio Frequency (RF) channel in the WPT system make possible to directly interact with events in the physical world, i.e. to monitor and collect data from physical processes of charging EV battery.

\section{WPT Simultaneously with the Wireless Data Transmission}

The wireless power and data transmission system with resonant coils tuned at one single working frequency is analyzed in [6-8]. The transmitted working frequency is modulated at the same time by the data transmission. In order not to be influenced by the 
energy transfer the data transmission can be treated by different technical methods, e.g. amplitude shift keying (ASK), frequency shift keying (FSK) and phase shift keying (PSK) modulation.

The feasibility of the power and data receiver implementation applying amplitude modulation with a single antenna is proposed in [6]. The system of wireless power and data transmission, as described in [8], operates at a frequency of $13.56 \mathrm{MHz}$ and allows simultaneous transmission of energy and data at speeds up to $1 \mathrm{Mb} / \mathrm{s}$. The principle of operation is the modulation of the carrier frequency by the information signal.

However, the efficiency of energy transfer in this method is low because the transferred average power is reduced by the modulation signal. The modulation signal cannot be too low since must be protected from the energy transferred, i.e., noise.

The contactless power and data transmission system that uses two pairs of resonant coils is shown in $[14,15]$. The adopted solution is based not only on separated coils for power and information transmission, but also on specially shaped multiple frames. The special geometry of data coils reduces the mutual coupling between the power and data coils. This way the voltage induced by electromagnetic field of WPT system can be reduced.

In the systems generally described, the power and data transfer are transmitted through the near electromagnetic field which operates across relatively short distances, i.e., up to a few meters. Unfortunately, the near field operation limits the communication speed and energy transfer efficiency.

To overcome these limitations a more efficient power and data transfer system is proposed to implement. In this system a bidirectional communication between the transmitter and receiver is created. For the coordination and optimization of the transmitter and receiver of the WPT system, a set of microcontrollers is in development. It is assumed that this system will transmit not only high density energy but also high-density (high speed) data through RF data channels.
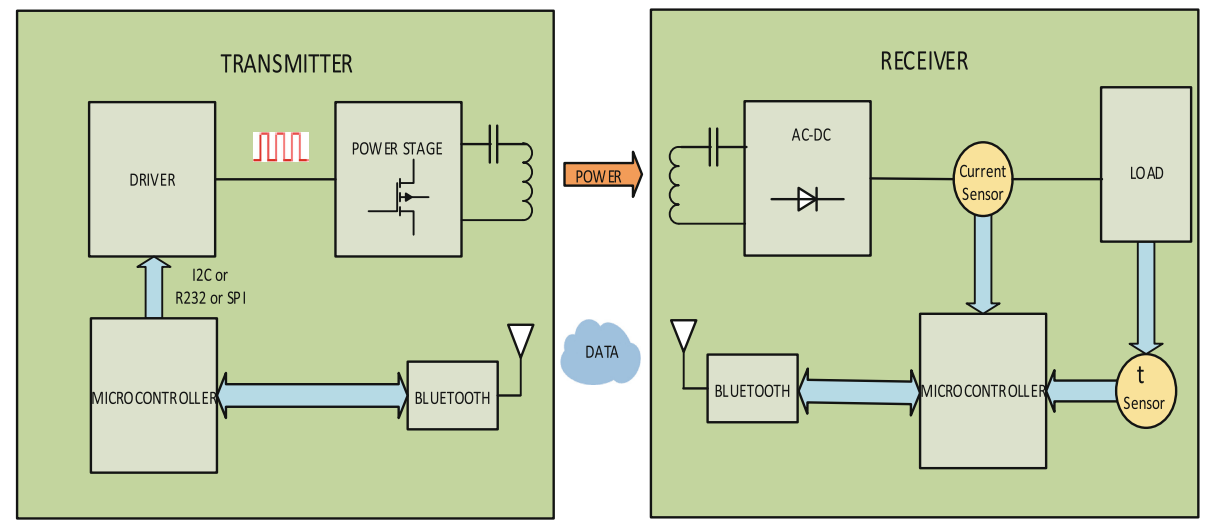

Fig. 1. Wireless Power Transfer System: Transmitter and Receiver Block Diagrams. 
Currently, in the Faculty of Science and Technology (FCT), University Nova wireless power and data transmission system based on magnetic resonant coupling are being constructed. The simultaneous wireless powering and data communication transmitter and receiver block diagram is shown in Fig. 1.

The system shown in Fig. 1 is based on magnetic resonant coupling. The transmitter consists of a driver, a power stage, a transmitting coil, and a microcontroller. The receiver side includes a receiving coil, AC-DC rectification, current and temperature sensing and another microcontroller. The load is the EV battery.

The circuit representation of the two-coil WPT system is shown in Fig. 2. The schematic is composed of two resonant circuits corresponding to the two coils. These coils are connected together via a magnetic field, characterized by coupling coefficient $k_{12}$. The coupling coefficient $k_{12}$ is given by:

$$
k_{12}=\sqrt{\frac{M_{12}}{L_{1} L_{2}}}
$$

where $M_{12}$ is the mutual inductance between the coils with inductances $L_{1}$ and $L_{2}$.

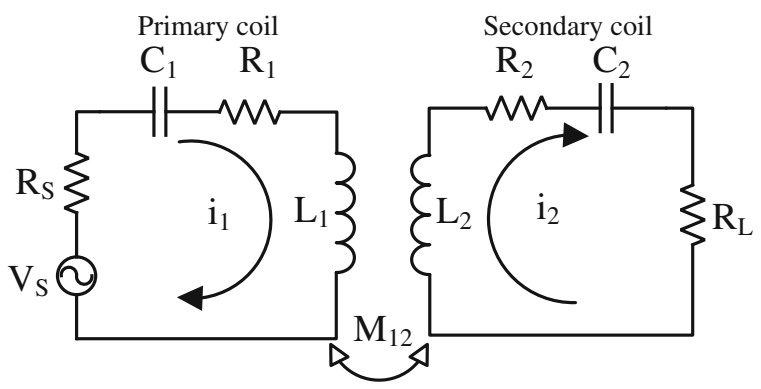

Fig. 2. The two-coil WPT system.

The loop impedances of the two coils are given by:

$$
Z_{1,2}=R_{S, L}+R_{1,2}+j \omega L_{1,2}+\frac{1}{j \omega C_{1,2}} .
$$

Using Kirchhoff's voltage law is given by:

$$
\begin{aligned}
& I_{1}\left(R_{s}+R_{1}+j \omega L_{1}+\frac{1}{j \omega C_{1}}\right)-j \omega I_{2} M_{12}=V_{S} \\
& I_{2}\left(R_{L}+R_{2}+j \omega L_{2}+\frac{1}{j \omega C_{2}}\right)-j \omega I_{1} M_{12}=0 .
\end{aligned}
$$




\section{Wireless Data Technology for WPT System}

A data transmission system with higher frequencies, i.e., on the order of $\mathrm{GHz}$ allows increasing the data transmission rate. In the case of exchange of information on advanced wireless transmission systems, several communication technologies could be adopted, such as Bluetooth, Wi-Fi, ZigBee [1, 9, 16].

The development and research using well known and well supported technology allows obtaining more reliable solutions. So one of important factors for the wireless data technology will be the high level of standardization and interoperability between devices from different manufacturers. According to [17], in general, there are five potential wireless networks that are being considered for intra- and inter-vehicles applications, namely Bluetooth, UWB, RFID, ZigBee and Wi-Fi. However, only the Bluetooth was implemented in several vehicles in recent years and is now a widely used technology in many vehicles $[16,18]$.

ZigBee, UWB and Wi-Fi have not yet been implemented in all vehicles, and investigations in this field are still developing [17]. The ZigBee fills a gap provided by other technologies, including wireless sensor interconnection for the control. It is expected that the ZigBee can be used in monitoring and control applications, related to temperature and moisture measurement, as well as heating, air conditioning and lighting control [19].

In order to generalize the communications in WPT systems, it is of any interest to use a well-known, developed and supported technology. In this way one of the important factors for choosing the wireless data transmission technology is the high level of standardization and interoperability between devices from different manufacturers [19].

Given these considerations and the fact that Bluetooth technology is widely used in the automotive industry $[17,19]$ it's considered a good compromise in terms of the data rate/efficiency/cost. According to these considerations, this technology was adopted since it is quite suitable for data transmission in WPT system.

\section{Experimental Results}

The analysis was concentrated on the EM radiation caused by converter operating at $20 \mathrm{kHz}$ switching frequency, at which the studied WPT system operates. In principle, the electromagnetic interferences from a relatively low frequency, as expected in case, probably will be not so strong. The Bluetooth technology operates at the frequency of $2.4 \mathrm{GHz}$ which is enough higher than the first harmonic of the power transfer frequency $(20 \mathrm{kHz})$. In order to verify this hypothesis some measurements and analysis were done.

The test equipment used to perform measurements was composed by an experimental setup of WPT system and the measurement system. The WPT system consists of a high frequency power source, transmitting resonant coil, receiving resonant coil and electronic programmable load. The measurement equipment consist of a basic unit, measuring instrument Narda SRM-3000 Selective Radiation Meter, 3-axis E-field antennas in $100 \mathrm{kHz}$ to $3 \mathrm{GHz}$ frequency domain and portable field strength meter, PMM 8053A.

The first series of measurements was taken in the FCT Laboratory. Some disturbance was observed at the frequency $1.9 \mathrm{MHz}$ which may be caused by the heavy consumption of nearby located smartphone. Because of this, in order to prevent 
external electromagnetic interference, the second series of measurements was made inside a Faraday cage. The experimental setup of WPT system and the measurement equipment in the Faraday cage are shown in Fig. 3.

The first measurement of second data series was executed when the WPT system was non-energized. The electric field in the absence of interference of the WPT system is shown in Fig. 4. As it may be observed, there are interferences at frequencies between $100 \mathrm{kHz}$ and $200 \mathrm{kHz}$. This can be explained by the nearby fluorescent lamp, whose electronic ballast was working. The electronic ballast operates in the range of frequencies from $25 \mathrm{kHz}$ to $133 \mathrm{kHz}$.

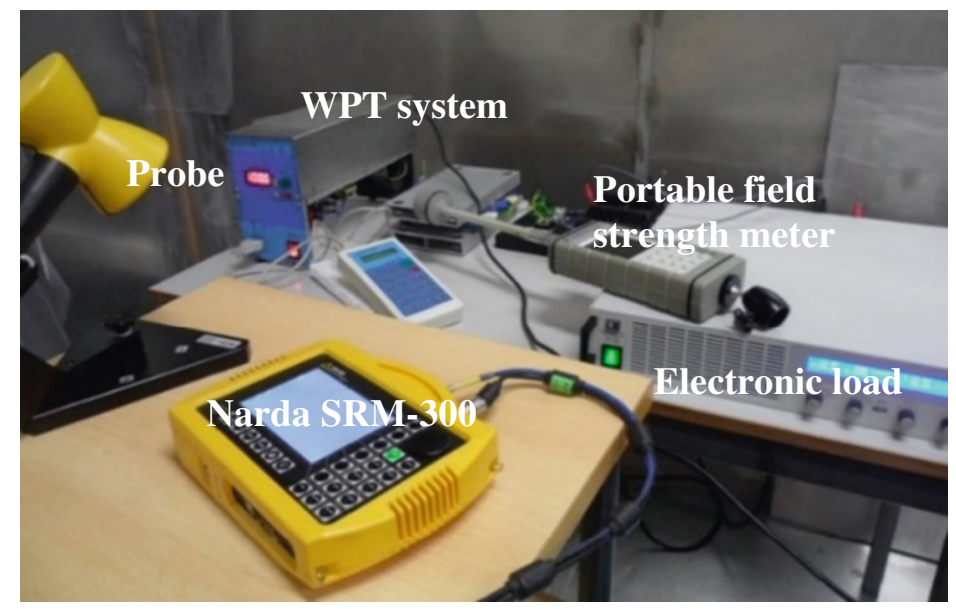

Fig. 3. WPT system - Experimental setup.

The harmonic generation from WPT system operating at $20 \mathrm{kHz}$ frequency is shown in Fig. 5. The used measurement equipment was capable to analyze frequencies starting from $100 \mathrm{kHz}$, so the amplitudes of the harmonics were measured starting from the 5th harmonic, corresponding to $100 \mathrm{kHz}$.

To obtain the 1st harmonic and the 3rd harmonic amplitudes, the minimum squares method was used. Using the method of minimum squares the experimental data were adjusted with a coefficient of determination $R^{2}=0.9685$. The high value obtained for $R^{2}$ value indicates that the trend line quite precisely fits the data. The amplitude of the harmonics including the 1st harmonic and the 3rd one generated by the WPT system is shown in Fig. 6.

In order to estimate the electromagnetic interference impact on data wireless channel, it is necessary to evaluate the bit error rate (BER), the packet error rate (PER), or throughput of the communication system.

The most usual method to evaluate receiving quality of data wireless channel, including the effect of interference and disturbance in industrial wireless communication, is to check the PER. The PER is the percentage of the number of packets that failed to be received correctly to the number of whole packets transmitted. The PER is also one of the factors determining system throughput and latency [20]. 


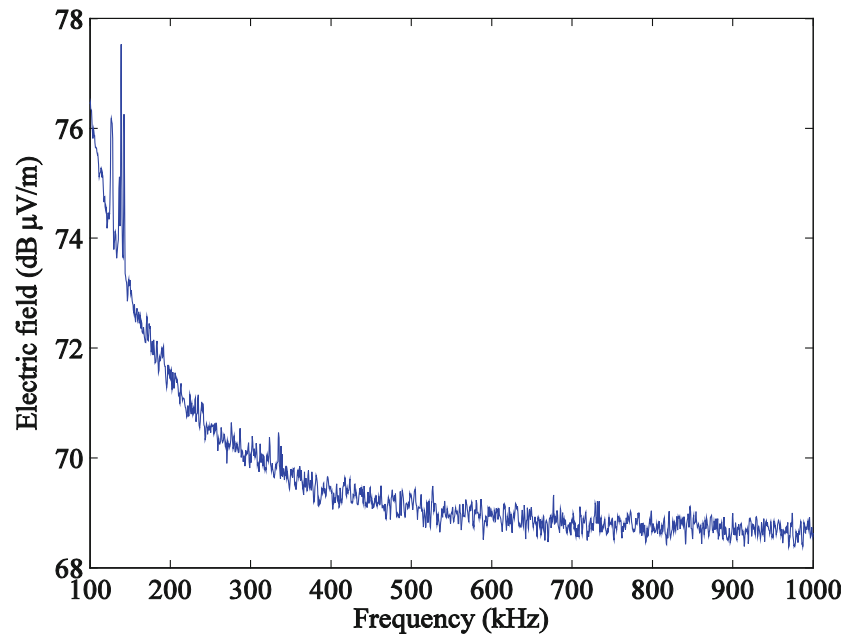

Fig. 4. Electric field in the absence of interference of the WPT system.

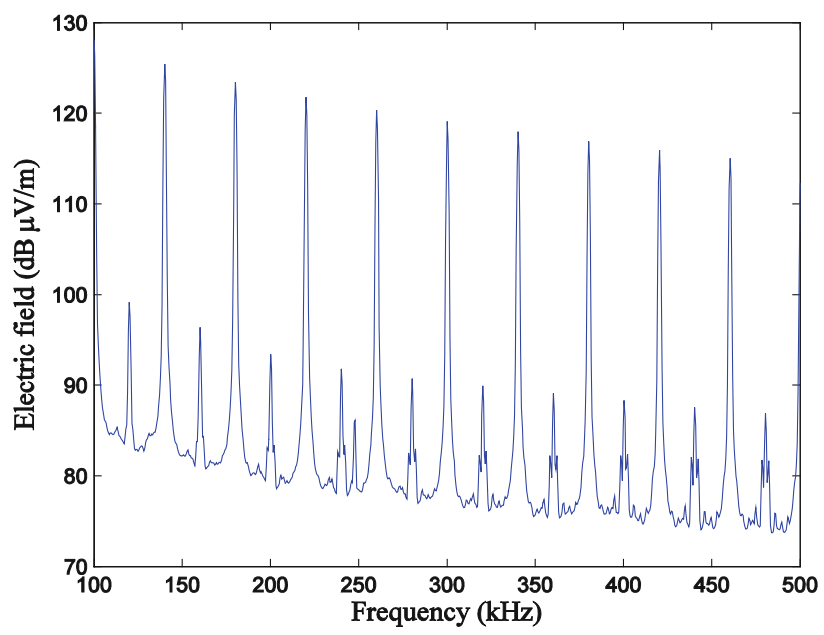

Fig. 5. Harmonic generation from the WPT system operating at $20 \mathrm{kHz}$ frequency.

The Bluetooth communication performance was evaluated using the free software Iperf3 [21] and Wireshark [22]. Initially Bluetooth communication between two laptops placed in the operating area of WPT system was established. One of the laptops was used as a server and another one as a client. The server created data streams and sent them to the client using the software Irerf3.

To capture frames and determine the PER a network protocol analyzer, Wireshark, was used. It can capture and analyze packets to determine those ones that were lost by transmission errors. 


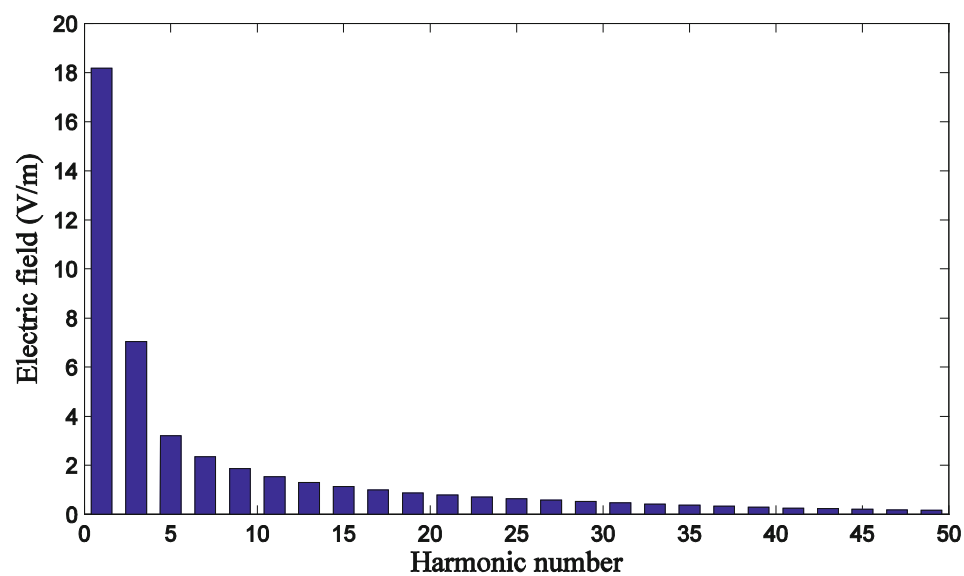

Fig. 6. Amplitude of the harmonics generated by the WPT system.

The results confirmed that there were no packets with transmission errors, so it can be considered that the studied WPT system doesn't influence the data wireless channel operating at $2.4 \mathrm{GHz}$ frequency.

\section{Conclusions}

This paper presents a study related to the electromagnetic interference impact of Wireless Power Transfer system on data wireless channel. The wireless data transmission is important to improve the efficiency of the wireless power transfer. The consideration about choosing the wireless data technology was also presented. From the described technologies, the Bluetooth can be considered one of the solutions for the data transmission in WPT systems.

To prevent external electromagnetic interference the measurements of the electromagnetic fields generated by WPT system were taken in the Faraday cage. The levels of electromagnetic fields due to the WPT system are present. In order to estimate the possible impact of the electromagnetic interference on data wireless channel the evaluation of packet error rate was made.

The results of experiments confirmed that there is no significant impact from Wireless Power Transfer system operating at $20 \mathrm{kHz}$ frequency on data transfer channel operating at $2.4 \mathrm{GHz}$ frequency.

\section{References}

1. Li, S., Mi, C.: Wireless power transfer for electric vehicle applications. IEEE J. Emerg. Sel. Top. Power Electron. 3(1), 4-17 (2015)

2. Valtchev, S.S., Baikova, E.N., Jorge, L.R.: Electromagnetic field as the wireless transporter of energy. Electron. Energ. Facta Universitatis, Nis, Serbia 25(3), 171-181 (2012) 
3. Kurs, A., Karalis, A., Moffatt, R., Joannopoulos, J.D., Fisher, P., Soljačić, M.: Wireless power transfer via strongly coupled magnetic resonances. Science 317, 83-86 (2007)

4. Imura, T., Okabe, H., Hori, Y.: Basic experimental study on helical antennas of wireless power transfer for electric vehicles by using magnetic resonant couplings. In: IEEE Vehicle Power and Propulsion Conference, pp. 9936-9404. Dearborn, USA (2009)

5. Chunbo, Z., Kai, L., Chunlai, Y., Ma, R., Hexiao, C.: Simulation and experimental analysis on wireless energy transfer based on magnetic resonances. In: IEEE Vehicle Power and Propulsion Conference, pp. 1-4. Harbin, China (2008)

6. Wu, C.M., Sun, J.S., Itoh, T.: A simple self-powered AM-demodulator for wireless/data transmission. In: 42th European Microwave Conference, pp. 325-328. Amsterdam, Holland (2012)

7. Tibajia, G.V., Talampas, M.C.R.: Development and evaluation of simultaneous wireless transmission of power and data for oceanographic devices. In: IEEE Sensors, pp. 254-257. Limerick, Ireland (2011)

8. Hmida, G.B., Ghairani, H., Samet, M.: Design of a wireless power and data transmission circuits for implantable biomicrosystem. Biotechnology 6(2), 153-164 (2007)

9. Yokoi, Y., Taniya, A., Horiuchi, M.,Kobayashi, S.: Development of kW class wireless power transmission system for EV using magnetic resonant method. In: 1st International Electric Vehicle Technology Conference, pp. 1-6. Yokohama, Japan (2011)

10. Obayashi, S., Tsukahara, H.: EMC Issues on Wireless Power Transfer. In: International Symposium on Electromagnetic Compatibility, pp. 601-604. Tokyo, Japan (2014)

11. Blaabjerg, F., Ionel, D.M.: Energy devices and systems - state-of-the art technology, research and development, challenges and future trends. Electr. Power Compon. Syst. 43(12), 13191328 (2015)

12. Foundations for innovation in cyber-physical systems - workshop summary report. National Institute of Standards and Technology (2013)

13. Batista, N.C., Melicio, R., Mendes, V.M.F.: Layered smart grid architecture approach and field tests by ZigBee technology. Energy Convers. Manage. 88, 49-59 (2014)

14. Bieler, T., Perrottet, M., Nguyen, V., Perriard, Y.: Contactless power and information transmission. IEEE Trans. Ind. Appl. 38(5), 1266-1272 (2002)

15. Rathge, C., Kuschner, D.: High efficient inductive energy and data transmission system with special coil geometry. In: 13th European Conference on Power Electronics and Applications. Barcelona, Spain (2009)

16. Wireless connectivity guide. Texas Instruments. www.ti.com

17. Green, R.J., Rihawi, Z., Mutalip, Z.A., Leeson, M.S.: Networks in automotive systems: the potential for optical wireless integration. In: 14th International Conference on Transparent Optical Networks, pp. 1-4. Coventry, UK (2012)

18. Woodings, R.W., Cypress, M.G.: Avoiding interference in the 2.4-GHz ISM Band. http:// www.eetimes.com/document.asp?doc_id=1273359

19. Ramteke, A., Gurmule, A., Sonkusare, K.: Wireless automotive communications. Discovery 18(53), 89-92 (2014)

20. Matsuzaki, M.: Reliability and stability of field wireless. Yokogawa Technical report English Edition, vol. 55:2, pp. 15-18 (2012)

21. ESnet: https://fasterdata.es.net/performance-testing/network-troubleshooting-tools/iperfand-iperf3/

22. Lamping, U., Sharpe, R., Warnicke, E.: Wireshark user's guide (2008) 